\title{
Das Haarkleid eines Fetus von Schimpanse
}

DORCH

\author{
Prof. L. BOLK (Amsterdam).
}

(Mit 8 Textfiguren).

In seiner ausführlichen Arbeit über die Richtung der Haare bei den Affenembryonen nebst allgemeinen Erörterungen über die Ursachen der Haarrichtungen, hat Schwalbe eine Fülle von Tatsachen und eine.wertvolle kritische Betrachtung über das Problem der Haarrichtungen geliefert. Leider war der Autor nicht in der Lage auch über Schimpanse Beobachtungen anzustellen, da fetales Material dazu fehlte. Das ist zu bedauern da, wie aus den vereinzelten sehr lückenhaften Litteratur-Angaben über die Haarrichtung bei Schimpanse schon zu schliessen war, bei diesem Anthropomorphen besonders vom menschlichen Zustand stark abweichende Verhältnisse vorliegen, und weiter die Angaben der Autoren über gewisse Punkte mit einander in Widerspruch sind.

Der Umstand, dass ich ein für die bezügliche Untersuchung sehr geeignetes Objekt besitze, veranlasste mich diese Lücke in unserer Kenntnis auszufüllen. Es betrifft den Fetus eines Schimpanse, dessen Haarkleid schon vollständig zur Anlage gelangt ist, die Haare sind aber noch so kurz, dass die Untersuchung nur mit Hülfe der Lupe geschehen konnte. Ehe ich zur Beschreibung meiner Befunde in den einzelnen Körperregionen übergehe, dürfen einige allgemeine Bemerkungen vorausgeschickt werden.

Wie gesagt war der ganze Körper schon behaart, die Haare waren aber noch sehr kurz. Schon makroskopisch war es aber sichtbar, dass an einzelnen Stellen die Haare eine grössere Länge erreicht hatten. Besonders interessant war in dieser Hinsicht der Kopf. In einer Ausdehnung, die ziemlich genau mit der behaarten Kopfhaut Jes Menschen úbereinstimmt, fand sich eine, aus ziemlich langen Haaren bestehende Kopfbekleidung. Diese Haare waren von einer kastanienbraunen Farbe, während die übrige Behaarung, so weit sie schon pigmenthaltig war, einen mehr schwarzen Ton aufwies. An zwei Stellen des Kopfes kamen Ausnahmen vor, und zwar an der Kinnhaut, und in der Regio superciliaris. Der Fetus besitzt nämlich eine gut ausgebildete Anlage von Bart und Augenbrauen, wovon man sich an Figur 1 überzeugen kann. In dieser Figur sind die Kopfhaare, der Deutlichkeit wegen nicht so lang gezeichnet als sie in Wirklichkeit sind, das Feld der langen Behaarung ist jedoch leicht zu erkennen.

Nun waren, wie gesagt, die Augenbranen schon kräftig entwickelt. Auffallend war die abweichende Farbe dieser Brauenhaare. Denn im Gegensatz zur kastanienbraunen Farbe der Kopfdachbehaarung, waren die Supercilien von einẹ schönen schwefel- oder kanariengelben Ton. Vereinzelt traten kurze Haare von der generellen Farbe zwischen diesen gelben Haaren auf.

Die Cilien besitzen die dunkle Farbe der Kopfhautbehaarung.

Eine zweite Stelle besonderer Gesichtsbehaarung war die Kinngegend. Auch diese war der Sitz von ziemlich langen Haaren, zarter als die Supercilien, mit welchen sie aber die leichte gelbe Färbung gemein hatten. 
Wir finden somit bei einem noch ziemlich jungen Schimpansenfetus eine Gesichts- und Kopfbehaarung, welche in gewissen Hinsichten Übereinstimmung mit dem Haarkleid des Menschen aufweist: ein stark behaartes Calvarium, Augenbrauen und eine Bart-Anlage. Die Tatsache, dass besonders die letztere aus helleren Haaren besteht als jene des Schädeldaches ist auch eine beim Menschen wohlbekannte, nicht selten auftretende Erscheinung. Bei einem jungen Schimpanse mit vollständigem Milchgebiss, den ich daraufhin untersuchte, traf ich zwar noch die längeren, hier weissen, Barthaare an, von gelben Brauenhaaren fand ich aber keine Spur mehr.-Weitere Besonderheiten, den Entwicklungsgrad der Haare betreffend, werde ich bei den einzelnen Körperteilen, zu welcher Beschreibung ich jetzt übergehe, geben.

Ich fange mit der Behaarung des Kopfes an, und verweise dazu auf Figur 1.

Aus Mangel an embryologischem Material hat Schwalbe die Haarrichtung bei einem jungen männlichen Schimpanse untersucht, und giebt davon die folgende Beschreibung (l. c. S. 108).

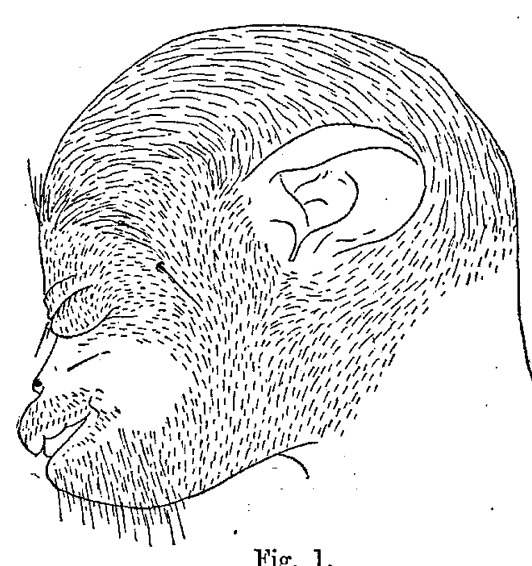
„Im Stirngebiet besteht eine mediane Scheitelung der Haare „ähnlich wie beim Gibbon, welche nach hinten etwa bis zur „Transversalebene der Ohrmitten reicht. Von diesem Scheitel "aus divergieren die Haare lateral zur Wange, zum Ohr und „zu den Seiten des Hinterhauptes. Entsprechend dem distalen „Rande des Unterkiefers treffen die schräg aufsteigenden Haare "des Halses auf die Haarē des Unterlippen- und Unterkiefer"gebietes, welche in der Nachbarschaft der Mundspalte dieser „zugekehrt sind, sodann in der entgegengesetzten Richtung „immer mehr sich aufrichten, um entsprechend der distalen "Grenze des Unterkiefers sich distal den aufsteigenden Hals„haaren entgegen zu neigen. Die Haare der Oberlippe haben „in der Mitte eine absteigende Richtung zur Mundspalte, die "seitlich immer mehr in die rein laterale Richtung übergeht".

Diese Beschreibung ist nicht vollständig und stimmt nicht ganz mit meinen Befunden überein, wie schon aus Vergleichung, der Schwalbe'schen Beschreibung mit der Figur 1 ersichtlich ist. Nur für die Oberlippe kann ich den Angaben des genannten Autoren beistimmen. Es sei hierzu jedoch bemerkt, dass gerade am Übergang vom behäarten in das unbehaarte, die Mundspalte begrenzende Gebiet der Lippe eine regelmässig angeordnete einfache Reihe von etwas strafferen Haaren eingepflanzt war. Seitlich stösst das behaarte Gebiet der Oberlippe an jenen Teil der Wangengegend, den ich in Anschluss an die ganze Regio nasalis noch völlig unbehaart fand. Nur ein einziges straffes Tasthaar in der Regio paranasalis zeichnete diesen Bezirk aus.

Die Beschreibung der allgemeinen Behaarung des Kopfes kann am besten Ausgang nehmen von dem durch eine tiefe Furche begrenzten Unterrand der unteren Palpebra. Das ganze Augenlid war besetzt mit feinen Härchen, die sagittal gestellt und nach oben gerichtet waren. Seitlich neigten sie einer mehr transversalen Richtung zu. Diese Haarströmung setzt sich auf das obere Augenlid fort. Nur die kräftigen Cilien des oberen Lides waren in gerade entgegengesetzter Richtung eingepflanzt. Ich stimme somit Schwalbe bei, wenn er, entgegen den Angaben Friedenthals darauf hinweist, dass die Augenlider wohl behaart sind. Diese rein sagittale Anordnung der Haare setzt sich über die ganze mediane Region der Stirn und des Calvarium fort. Die von Schwalbe beschriebene mediane Scheitelung, konnte ich an meinem Individuum nicht feststellen, in und neben der Medianlinie waren die Haare über den ganzen Kopf rein sagittal eingepflanzt. In der seitlichen Stirngegend schlugen die Haare eine mehr transversale Richtung ein und in der Temporalgegend biegen sie sich immer mehr nach unten. In der Mitte der genannten Gegend stösst diese Haarströmung zusámmen mit einer die an der Implantationslinie der Ohrmuschel anfängt. Am oberen Ende dieser Linie waren die Haare fast rein horizontal eingepflanzt, die Richtung ändert sich aber sehr schnell, sodass vor dem Tragus die Haare sagittal nach unten gerichtet sind. Die von der Stirn und von der Ohrmuschel kommenden einander entgegengesetzten Haarzüge, gehen in 
der unteren Temporalgegend allmählig in einander über, sodass die Wange und der grösste Teil der Mandibulargegend mit etwas schräg nach unten und vorn gerichteten Haaren besetzt sind. Der Vorderrand dieses Gebietes bildet die Begrenzung des haarfreien Bezirkes. Dieser Rand fängt unter dem unteren Augenlid an, woselbst die Haare rein transversal implantiert sind um lateralwärts bald in die vertikale Richtung umzubiegen. Seitlich vom Auge fand sich beiderseitig ein kräftiges Sinushaar.

Die untere Lippe und die Kinngegend sind mit den schon beschriebenen langen Barthaaren besetzt welche mehr sagittal eingepflanzt sind als jene der seitlichen Unterkiefergegend. Bemerkenswert ist eine vom generellen System abweichende Behaarungszone der Unterlippe, welche dem schleimhautartigen Lippensaum entlang sich erstrecke, und die mit kurzen, aber straffen Haaren besetzt war, welche der Mundspalte entgegengerichtet sind.

Unmittelbar vor dem unteren Ende der Ansatzlinie des Ohres erstreckt sich eine kurze Konvergenzlinie. Wie schon gesagt sind vor dem Tragus die Haare vertikal nach unten gerichtet. Verfolgt man nun den von der Kopf haut kommenden Haarzug, dann sieht man, dass dieser das $\mathrm{Ohr}$ umkreist, sodass schliesslich die Haare sagittal und nach oben gerichtet sind. Letztere stossen dann an den vor dem Ohre, nach unten verlaufenden Haarzug.

Es ist nicht mein Absicht in dieser Abhandlung eine Vergleichung des Haarkleides von Schimpanse mit jenem der anderen Anthropomorphen oder. des Menschen anzustellen, ich wünsche mich der Hauptsache. nach auf eine einfache Beschreibung meiner. Befunde zu beschränken. Wir setzen daher unsere Beschreibung mit der Betrachtung des Haarkleides an Hals und Rumpf fort.

Wir knüpfen unsere Beschreibung. zunächst an Figur 2 an, welche eine Übersicht über die Haarzüge von Hals und Rumpfe in ventraler Ansicht gestattet.

Die schräg nach unten und vorn gerichtete Haarströmung. im subauriculären Gebiet beschrieben setzt sich über die seitliche Fläche des Halses fort. Wenn diese Strömung die vordere Halsfläche erreicht, ändert sich die Haarrichtung allmählig, zuerst in eine mehr horizontale, und sodann in eine von unten lateral nach oben medial gerichtete. Je mehr die Haare der Medianlinie genähert sind, desto mehr richten sie sich nach oben, sodass in der Medianlinie eine Konvergenzlinie entsteht, wobei die beiderseitigen Haarzüge einen kaudalwärts offenen Winkel bilden. Im submentalen Gebiet wird dann eine transversal verlaufende Konvergenzlinie gebildet, zwischen den vom Halse kommenden nach oben gerichteten Haaren, und den nach unten gerichteten der Kinngegend.

In der unteren Hälfte des Halses, spaltet sich von dem beschriebenen Haarstrom einer ab, in dem die Haare die Richtung die sie an der Seitenfläche des Halses haben, beibehalten, das heisst schräg von oben lateral nach unten medial gerichtet sind. Auch von dieser Strömung richten sich die Haare mehr

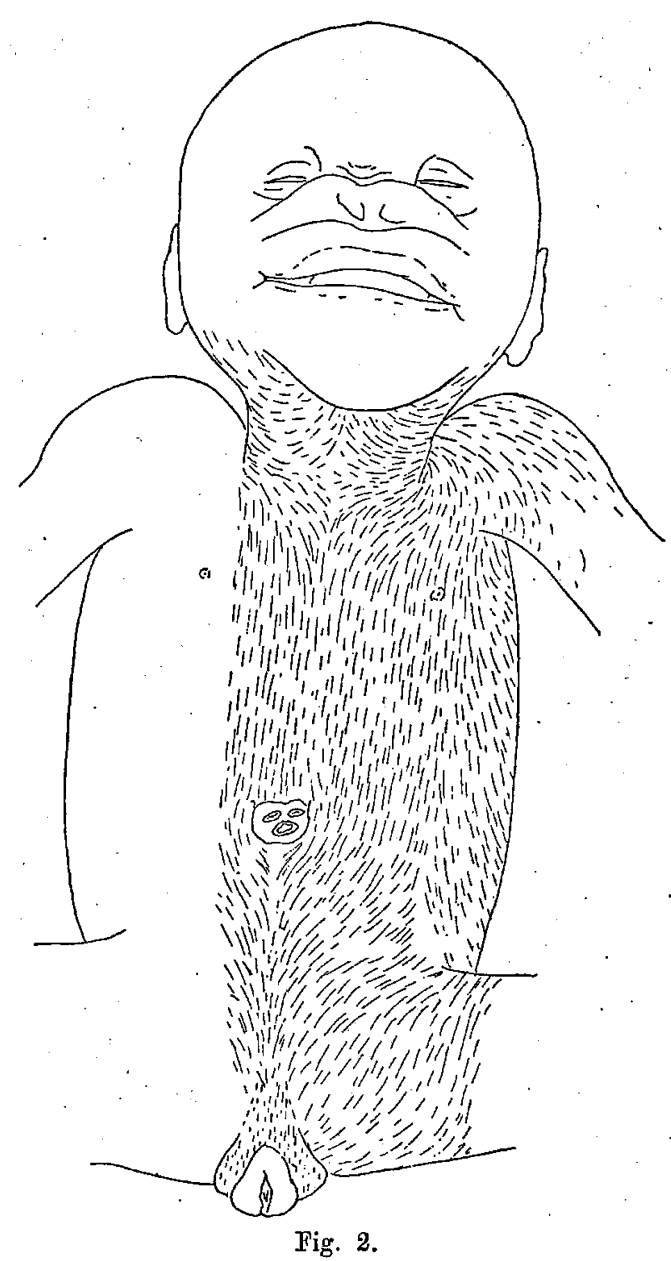

Fig. 2. kranio-kaudal, je näher sie der Medianlinie implantiert sind. Indem der Haarstróm im oberen Teil des Halses einen nach oben offenen Bogen beschreibt, im unteren einen mehr kaudalwärts konkaven, wird an der Übergangsstelle von Hals und Rumpf ein Kreuz gebildet, das sogenannte Sternalkreuz. Dasselbe ist ein Konvergenzkreuz, in Gegensatz zu dem von Schwalbe bei Macacus cynomolgus an übereinstimmender Stelle beschriebenen, das ein Divergenzkreuz war. 
Anch meine Befunde am Halse, stimmen nicht ganz mit den Angaben Schwalbe's überein. Der genannte Autor äussert sich darüber folgender Weise (l. c. S. 108): „In ähnlieher Weise wie beim Gibbon divergieren von der Einsenkung zwischen Schulter und Seitenfläche des Halses die Haare nach vorn und oben zur Seitenfläche des Halses, sodass man die Richtung der Haare am Halse als schräg von lateral nach median oben bezeichnen kann". An meinem Object trifft diese Richtung nur für den vorderen oberen Teil des Halses zu, und ein Ausstrahlungszentrum seitlich im unteren Gebiet des Halses fand ich nicht. Der Haar-

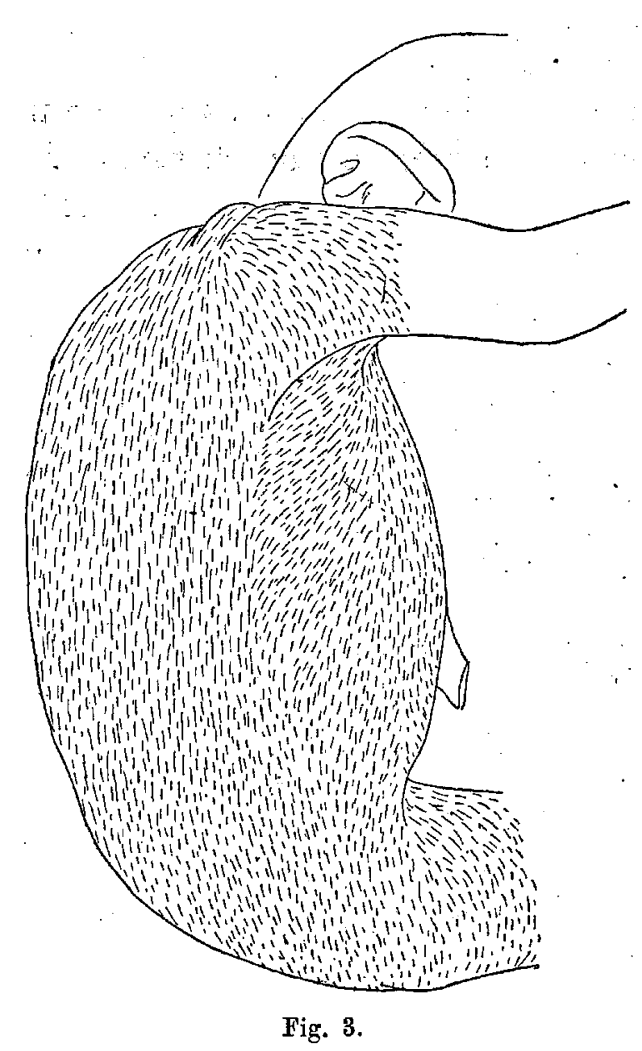
strom an der Seitenflache des Halses ist die Fortsetzung jenes der Seitenteile des Hinterhauptes. Von dem an meinem Object so schön entwickelten Sternalkreuz macht weiter Schwalbe keine Meldung. Im Nacken sind die Haare, die Richtung jener der medialen Zone des Hinterhauptes fortsetzend, sagittal implantiert, der Haarstrom zieht in kaudaler Richtung.

Der Rumpf ist in bezug auf die Haarzüge bequem in drei longitudinale Felder einzuteilen, ein ventrales, laterales und dorsales; wovon man sich leicht mit Hülfe von Figur 2 und 3 überzeugen kann.

In das dorsale Feld, den ganzen Rücken umfassend, waren die Verhältnisse sehr einfach. Als Fortsetzung der Nackenbehaarung kommt über den ganzen Rücken ein sagittaler Strom vor, wobei die Haare in kraniokaudaler Richtung implantiert sind. Im oberen Teil des Rückens waren die Haare am längsten, kaudalwärts wurden sie immer kürzer. Ein medianer Streifen, über das Sacrum sich erstreckend, war noch völlig unbehaart, wie aus Figur 4 ersichtlich. Hier war die Haut der Länge nach ein wenig narbig eingezogen.

Seitlich dehnt: das dorsale Feld sich bis zur hinteren Axillarlinie aus. Im oberen Teil ist diese Grenzlinie in Folge des Zusammenstossens mit einer entgegengesetzten Haarströmung scharf markiert, kaudalwärts wird die Grenze zwischen dorsalem und lateralem Haarfeld immer undeutlicher, um schlieszlich gänzlich verwischt zu werden (Vergl.. Fig. 3).

Das ventrale Feld ist etwas mehr kompliziert. Oben fängt es in der Claviculargegend mit dew unteren Halshaarzug an, in dem die Haare latero-medialwärts gerichtet sind, um über den Thorax bald in eine rein sagittale Richtung überzugehen. Seitlich erstreckt sich dieses Feld bis zur vorderen Axillarlinie. Die Papilla mammae hat gar keinen Einfluss auf die Haarrichtung, es bleibt dieselbe bis zur Nabelebene eine rein sagittale. In der Regio subumbilicalis schlagen die Haare eine medio-kaudale Richtung ein, wodurch eine mediane Konvergenzlinie entsteht, wobei jedoch die in, und unmittelbar neben der Medianlinie implantierten Haare wieder ziemlich rein sagittal gerichtet sind.

Durch die mediale Abbiegung der Haare in der subumbilikalen Gegend entsteht im unteren Teil des Rumpfes, unmittelbar oberhalb der Extremität, eine Divergenzlinie, welche nur bis zur Ebene des Nabels zu verfolgen ist. Diese Linie grenzt ventraler und lateraler Feld von einander $a b$.

Das letztgenannte Gebiet ist oben am schärfsten abgegrenzt, da es hier zwischen der vorderen und hinteren Axillarlinie gefasst ist. In diesem thorakalen. Teil des Gebietes sind die Haare schräg von oben vorn nach. unten und hinten eingepflanzt (Fig. 3).

Daher entsteht eine vordere Divergenzlinie und eine hintere Konvergenzlinie als Begrenzung. Da sich in der Bauchgegend die Haare immer mehr sagittal und zwar kraniokaudal richten, verschwindet allmählig die hintere Begrenzungslinie, da, wie beschrieben, im Rückenfeld der Haarzug ebenfalls ein kranio-kaudaler ist. Die vordere Begrenzungslinie 
dagegen ist vollständiger, da im ventralen Haarfeld subumbilikal die Haare von der sagittalen in einer mehr schrägen Richtung abbiegen.

Im ganzen genommen weist folglich das Haarkleid des Rumpfes ( - über das kaudale Ende wird später noch gesondert gehandelt-) sehr einfache Haarzüge auf. Die Haare sind generell kraniø-kaudal implantiert, nur eine subaxilläre und eine subumbilikale Zone zeigt Abweichung von diesem allgemeinen Schema.

Meine Befunden sind in Widerspruch mit den Abbildungen, welche Duckworth von der Schimpansebehaarung am Rumpfe giebt. (Die bezüglichen Figuren sind von Schwalbe als Textfigur 21 and 22 seiner Arbeit kopiert). Nach Duckworth würde nur ein dorsales und ventrales Feld vorkommen, durch eine vollständige, vom Halse bis zur Inguinalgegend sich erstreckende Divergenzlinie von einander getrennt. Im vorderen Gebiet, dessen Begreñzung an der Brust, ungefähr mit der Papillarlinie übereinstimmen sollte, würden die Haare schräg von oben lateral nach unten und medial gerichtet sein, im Rückenfeld schräg von oben vorn nach unten und hinten. Ich kann nicht glauben, dass hier eine richtige Beobachtung vorliegt; zumal auch Schwalbe dieselben nicht bestätigen konnte, dagegen Angaben macht, welche besser zu den meinigen stimmen. Er schreibt über die Rumpfbehaarung S. 109, l. c.: „es war bestimmt festzustellen. dasz an der ganzen ventralen Seite des Rumpfes bis zum Nabel und ebenso an den Seitenflächen desselben eine longitudinale kranio-kaudale Richtung bestand, wie an der dorsalen. Seite des Rumpfes. Eine Störungsstelle deren Art aber nicht genau zu ermitteln war, findet sich zweifellos im Axillargebiet; eine inguinale Störungslinie mit Divergenz der Haare einerseits nach der unteren Bauchregion andererseits nach Vorderfläche des Oberschenkels war besser zu konstatieren." Wenn man in Betracht zieht, dass Schwalbe seine _Untersuchung an einem jungen Tier mit schon langen Haare anstellte dann ist es leicht verständlich, dass seine Beschreibung nicht so detailliert sein konnte, als die von mir gegebene. Wie man aber ersieht stimmen in groben Zügen unsere Befunden mit einander überein.

Das kaudale Rumpfende erheischt eine gesonderte Besprechung, für die ich Figur 4 zu Grunde lege.

Wie schon bemerkt worden ist, erstreckt 'sich über das Sacrum eine noch haarlose Stelle, die in der Mittellinie der Länge nach etwas narbig eingezogen, sich bis zum noch stark hervorragenden Steiszbeinhöcker erstreckt.

Die Analöffnung nimmt die Mitte eines kreisrunden haarlosen Bezirkes ein; der be. grenzt wird durch einen Kranz zur Analöffnung konvergierend eingepflanzter Haare.

Das stark hervorragende äussere Genital, durch die, die Schamspalte begrenzenden Labia minora dargestellt, ist völlig haarlos. Schon am anderer Stelle ${ }^{1}$ ) habe ich darauf aufmerksam gemacht, dass beim fetalen Schimpanse deutlich eine Anlage von Labia majora und Mons veneris zu sehen ist. Beim erwachsenen. Weibchen dagegen, ja sogar schon beim noch infantilen Individuum, ist diese Bildung verschwunden. Aus Figur 2 und 4 ist das Vorkommen und die Ausbreitung desselben zu ersehen. Dass man hier wirklich mit einer besonderen Region der Oberfläche zu tun hat, geht nun weiter aus der Behaarung hervor. Denn das bezügliche Gebiet stellt ein besonderes Haarbezirk dar, in dem die Haare rein sagittal ein. gepflanzt sind. Am Bauche anfangend sind die Haare von oben nach unten gerichtet, der Krümmung des Feldes nach hinter folgend, biegen sie in einer nach vorn und hinten schauenden Richtung ab.

Die Behaarung der Perinealgegend: war sehr eigentümlich (Vergl. Figur 4). Der, den Anus umkreisende Haarkranz, war vollständig, und durchzog dann auch die Medianlinie zwischen der genannten Öffnung und der Schamspalte. Jederseits der Medianlinie, fand ich

1) Über das kaudale Rumpfende eines Fetus vom Schimpanse. Anatomischer Anzeiger, Bnd L. S. 354, Jena 1917. 
eine nahezu kreisrunde völlig nackte Stelle. Jede desselben war umgrenzt von einer schmalen Haarzone, in der die Haare radiär angeordnet, wie von dem Zentrum der Stelle auszustrạhlen scheinen. Dadurch wird der ano-perineale Bezirk der Sitz eines Haarkranzes mit einstrahlenden Haaren um den Anus, und zweier Kränze mit ausstrahlenden Haaren, seitlich von der perinealen Mittellinie. Letztere fliessen ventral vom Anus in der Medianlinie mit einander zusammen.

Die Bedeutung der beiden perinealen haarfreien Stellen darf vielleicht darin gesehen werden, dass es hier eine Reminiscenz an die ebenfalls völlig haarfreien Gesässschwielen niedriger Affen gilt. Bei menschlichen Embryonen ist davon bisher keine auch nur.vorüber-

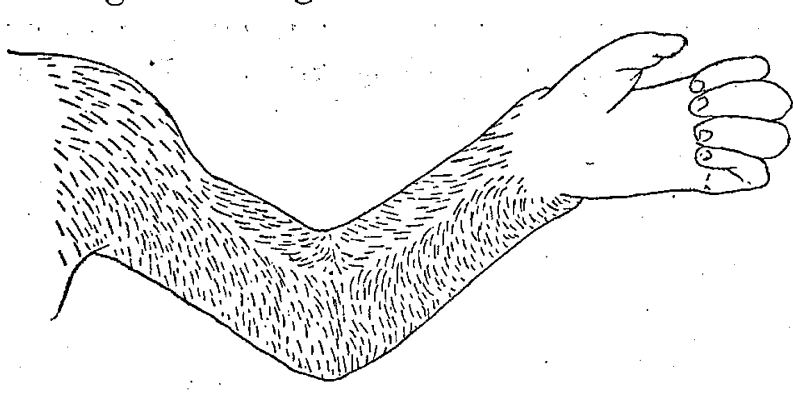

Fig. 5. gehende Andeutung gefunden. Ich weise aber darauf hin, dass das kaudale Rumpfende vom Schimpanse im allgemeinen beim Fetus mehr primitive Merkmale aufweist als es beim Menschen der Fall ist. Der noch deutlich hervorragende Steissbeinhöcker, die noch stark nach hinten sehende Genitalien sind ein Paar solcher Merkmale. Beim jugendlichen Individuum sind die bezüglichen Stellen behaart.

Über die Verhältnisse der Haarrichtungen an den Extremitäten können wir uns kurz fassen, denn sie weichen wenig von den bei Affen sonst bekannten Verhältnissen ab. Wir fangen mit der Betrachtung der oberen Extremitat an, unter Hinweis auf die Figuren 5 und 6.

Es zerfällt das Haarkleid der oberen Extremitat deutlich in drei Bezirke, nahezu mit den drei Abteilungen des Gliedes, Oberarm, Unterarm und Hand übereinstimmend. Die Haarströmungen des Oberarmes und der Schulterwölbung sind als Fortsetzungen jener der ventralen und dorsalen Fläche des Rumpfes zu betrachten. Auf der Schulterwölbung sind die Haare ziemlich rein in longitudinaler Richtung eingepflanzt, das heisst der Längs-

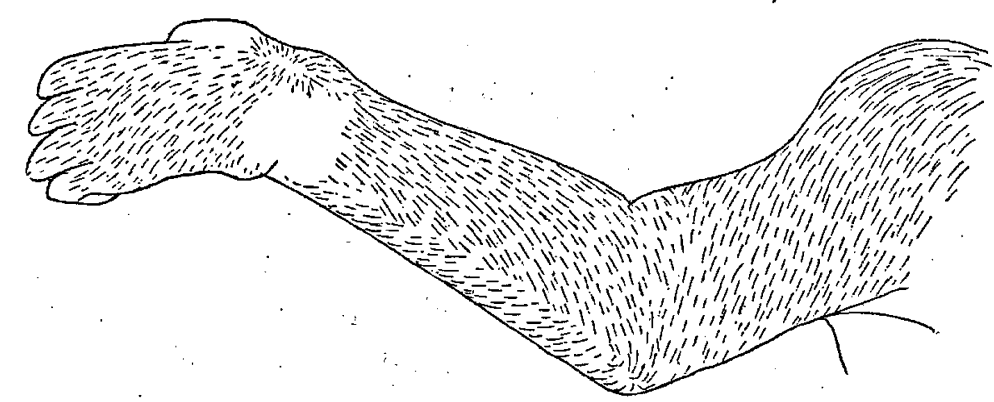

Fig. 6. achse der Extremität parallel. In der Richtung der Axillarböhle aber stellen die Haare sich mehr transversal zur Längsachse. Ungefähr zur Höhe der Insertion des Deltoïdes, treten zwei Linien auf, und zwar eine sehr deutliche Divergenzlinie welche an der ventralen Fläche des - Oberarmes nahe am radialen Rande verläuft und die in Figur $\mathbf{5}$ sehr scharf ausgeprägt erscheint, und eine Konvergenzlinie, die jedoch viel weniger deutlich ist als die erstgenannte, und bezüglich deren Auftreten man aus Kombination der in Figur 5 und 6 gezeichneten Verhältnisse schliessen kann. Diese Linie folgt nahezu den Ulnarrand des Oberarmes. Von der ventro-radial gelagerten Divergenzlinie strahlen die Haare in schräger proximo-distaler Richtung aus, bis zum Ellbogen und stossen hier auf den vom Unterarm kommenden Haarstrom. Dieser bildet wie gesagt ein Gebiet für sich. Man kann sagen, dass im allgemeinen der Haarstrom des Unterarmes jenem des Oberarmes entgegengesetzt ist.

Die Divergenzlinie der ventralen Fläche setzt sich; wie aus Figur $\mathbf{5}$ ersichtlich, vom Oberarme auf den. Unterarm bis zum Handgelenk fort. Die Haare aber kebren ziemlich plötzlich beim Übergange auf den Unterarm ihre Implantionsrichtung um, sie strahlen von der Divergenzlinie schräg in disto-proximaler Richtung aus. Dadurch wird das auch bei andren Anthropoïden auftretende Ellbogenkreuz gebildet. Von diesem Zentrum geht eine Konvergenzlinie in transversaler Richtung aus, welche ziemlich wohl mit der Biegungslinie der Extremität im Ellbogengelenk zusammenfällt, und wie gürtelförmig die ganze Extremität umkreist.

Die beiden divergenten Haarzüge setzen sich von der ventralen Fläche des Unterarmes, 
den medialen resp، radialen Rand herum auf die dorsale Fläche fort. Die Stromrichtung bleibt hier im grossen und ganzen die nämliche, es sind die Haare in disto-proximaler Richtung eingepflanzt, mehr oder weniger schräg, wie aus Figur 6 ersichtlich. Dadurch wird auf die dorsale Fläche des Unterarmes, dem Ulnarrande genähert, eine deutliche Konvergenzlinie gebildet. Es verläuft diese nicht in einer Flucht mit jener am Oberarme, die übrigens viel weniger scharf ausgesprochen ist, wie schon bemerkt wurde.

Dem Ellbogenkreuz an der Ventralseite entsprechend, entsteht an der Dorsalseite - und zwar zusammenfallend mit der Olecranonspitze, ein Konvergenzzentrum, in dem die Haare spiralförmig angeordnet sind.

Der Übergang von Unterarm auf Hand ist an der Dorsalseite durch eine haarloose Stelle gekennzeichnet, sodass das Haarsystem der Hand eine Selbständigkeit erlangt. Es ist derselbe sehr merkwürdig.

Proximal vom Daumen findet sich auf den Handrücken ein schön entwickeltes Divergenzzentrum, von wo die Haaren kranzartig ausstrahlen. Nur distal vom Handgelenke breitet sich dieser Kranz zu einem Haarzug aus, der den Handrücken überzieht. Die Haarrichtung ist im allgemeinen eine proximo-distale, wobei aber die Haare sich schräger stellen je mehr sie dem Ulnarrand der Hand näher eingepflanzt sind.

Von dem Handrücken greift die Behaarung auf die Finger über. Der Daumen ist noch völlig unbehaart, und von den übrigen Fingern waren ebenfalls der Mittel- und Endphalanx noch haarfrei, auf den Grundphalangen sind die Haare proximo-distal gerichtet.

Meine Befunde bezüglich des Haarkleides der oberen Extremität, zeigt manches Übereinstimmendes mit den immerhin kurz gefassten Angaben von Schwalbe. Dieselben beziehen sich aber nur auf die Dorsalseite der Extremität. Auch Schwalbe weist auf die an einander entgegengesetzten Haarrichtungen von Ober- und Unterarm hin, und memoriert das Konvergenzzentrum am Ellbogen. Es ist damit wohl das dorsale gemeint, da die Ventralseite nicht untersucht werden konnte. Es bleibt dann auch die Divergenzlinie an der Ventralseite und das darin gelegene ventrale Konvergenzzentrum unerwähnt. Die Beschreibung der Haarrichtung am Handrücken, und besonders das Auftreten des radialen Handzentrum stimmen mit meinen Befunden sehr gut überein, allein es erwähnt der Autor nicht die haarloose Stelle über dem Handgelenk. Die Ursache davon liegt wohl in den verschiedenen Alter der untersuchten Individuen. Denn, wie ich mich habe überzeugen können, an einem jugendlichen. Individuum, wird später auch diese Stelle vom Haarkleid überzogen. Warum an dieser circumscripten Stelle der Durchbruch der Haare verzögert ist, ist mir rätselhaft. Vielleicht ist die Lösung dieser Frage in jener Richtung zu suchen, dass die Behaarung von Hand und Unterarm, an zwei Stellen, unabhängig von einander anfängt, wobei die behaarten Felder einander allmählig nähern. Die Ausbreitung des Haarkleides würde dann nicht regelmässig in proximo-distaler Richtung sich fortsetzend, vom Unterarm auf die Hand übergreifen, sondern der Handrücken würde ein selbständiges. Territor darstellen, das sekundär mit jenem des Unterarmes zusammenfliesst.

Auch Schwalbe weist auf die Möglichkeit hin, dass die Haarentwicklung nicht regelmässig in distaler Richtung fortschreitet. Er gründet diese Ansicht auf seine Beobachtung dass bei einem Fetus von Macacus fuscatus die Haare am Handrücken stärker entwickelt sind als am Ober- und Unterarm.

Ich möchte die Frage stellen, ob dieses Prinzip: Es żerfällt der Haut in verschiedenen Territorien, und jedes Territor ist Zentrum einer selbständigen Haarkleidanlage, nicht als Erklärungsversuch der Entstehung von Konvergenz- und Divergenzlinien verwendet werden kann. Solche Linien und Zentra würden dann die Marklinien sein, längs welchen die selbständigen Haarkleidanlagen einander begegnen, etwa wie die Nähte der Schädeldachknochen, während die Ausstrahlungszentra die Stellen bezeichnen wo die Anlage und Verbreitung Ausgang nahm, also etwa vergleichbar mit den Ossificationszentren der genannten Knochen. Es kommt mir vor dass diese Gedanke wohl verdient durch Untersuchung von Embryonen verschie. denen Alters auf ihre Richtigkeit geprüft zu werden. 
Wenden wir uns jetzt der unteren Extremität zu. Die Figuren $y$ und 8 veranschaulichen die an diesem Gliede gefundenen Verhältnisse.

Im ganzen zeigt das Haarkleid der unteren Extremität weit einfachere Verhältnisse als jenes der oberen Extremität, und zwar schon deshalb, weil der Gegensatz der Haarzüge

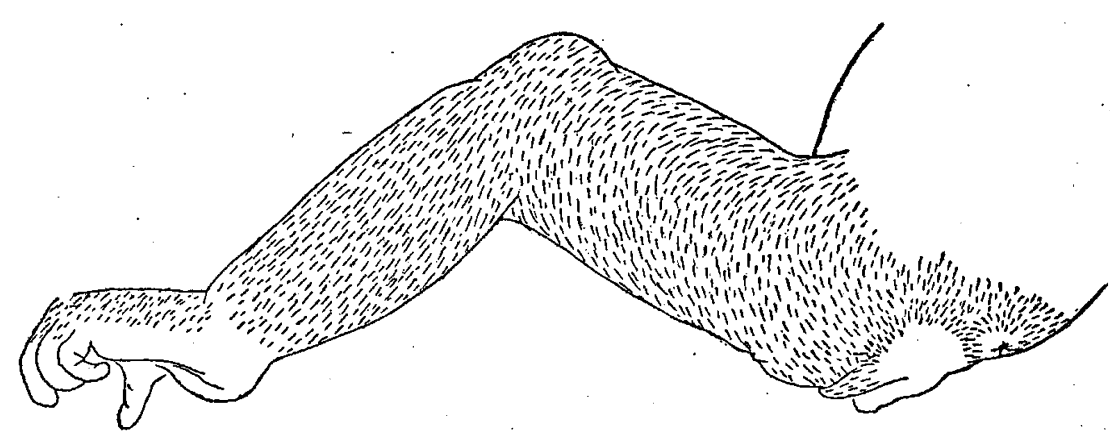

Fig. 7. zwischen Ober- und Unterarm, bei der unteren Extremität eigentlich kein Analogon hat. Es ist mehr eine einheitliche Haarströmung, welche die ganze Extremität überzieht. 'Es fehlen hier daher die Konvergenzund Divergenzlinien welche an der oberen Extremität so schön ausgeprägt waren.

Der Hauptsache nach ist die Haarrichtung eine longitudinale, wobei die Haare in proximo-distaler Richtung implantiert sind. Ausnahmen zu dieser allgemeinen Haarrichtung kommen nur an einem Teil des Oberschenkels und am Fussrande vor..

Auf der vorderen Fläche des Oberschenkels sind die Haare longitudinal in proximodistaler Richtung eingepflanzt. Auf der medialen Fläche (vergl. Figur 8) schlagen sie allmählig eine mehr transversale Richtung ein, der Haarzug ist hier ein bogenförmiger, und die Krümmung dieses Bogens ist so stark, dass auf der hinteren Fläche die Haare in disto-proximaler Richtung implantiert sind. Eine nämliche Anordnung zeigen die Haare auf der lateralen Seite des Oberschenkels (Figur \%). Eine Konvergenzlinie zwischen den Haarzügen der lateralen und medialen Fläche wird aber nicht gebildet, denn die Umbiegung des Haarstromes aus der proximo-distalen in der distoproximalen findet an beiden Seitenflächen des Schenkels so regelmässig statt, dass in einem ziemlich breiten Saum der Hinterfläche die Haare proximalwärts gerichtet sind.

Auf dem Unterschenkel sind die Haare ziemlich regelmässig in longitudinaler Rich-

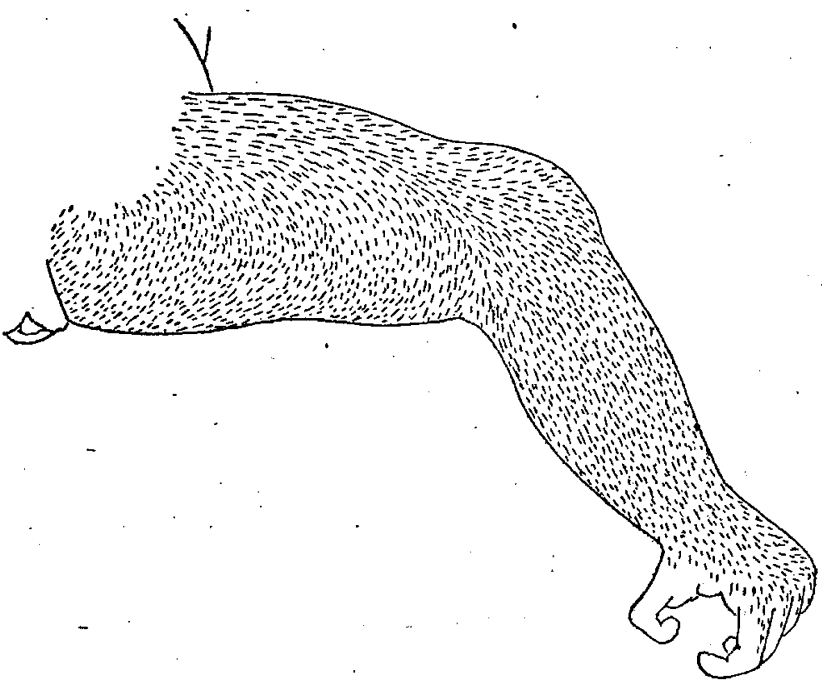

Fig 8. tung angeordnet, sie sind distalwärts gerichtet, und ohne Abänderung setzt sich der Haarzug vom Unterschenkel auf den Fussrücken fort, nur in den Randzonen wenden sich die Haare mehr medialwärts resp. lateralwärts. Von den Zehen waren nur die Grundphalungen behaart.

Es kommt. auf der ganzen unteren Extremität nur eine einzige Störungsstelle. vor, und zwar eine Divergenzlinie in der Kniekehle. Besonders sei darauf hingewiesen, dass ein. Divergenzzentrum am Fussrücken, wie es sso schön auf dem Handrücken ausgebildet war, völlig fehlt.

Meine Befunden am Haarkleid der unteren Extremität von Schimpanse, stimmen sehr gut überein mit der Beschreibung, welche Schwalbe von diesem Gebiet giebt. Nur spricht dieser Autor von einer Konvergenzlinie an der hinteren Seite des Obersehenkels, welche, wie oben erwähnt bei dem von mir untersuchten Fetus nicht da war, da die Härchen hier über einem mittleren Saum proximalwärts gerichtet waren. Unmöglich ist es aber nicht, dass in Folge des weiteren Wachstums dieser Saum immer schmäler wird, sodass schlies- 
lich eine Konvergenzlinie entsteht, $d a$ dann die von aussen und von innen kommenden Haarzüge in einen Winkel aneinanderstossen.

Hiermit möchte ich diese kurze Mitteilung abschliesen. Da es nur mein Zweck war, durch eine Beschreibung eine Lücke in unserer Kenntnis des Haarkleides einer uns so nahe verwandten Form zu geben, werde ich auf eine Vergleichung der beschriebenen Zustände bei Schimpanse mit jenen des Menschen oder der niedrigen Affen verzichten. Denn eine solche Vergleichung würde uns notwendig vor Fragen nach Entstehung der Erscheinungen und Erklärungsversuehen derselben - stellen, und dazu müsste ich über mehr eigene Erfahrung und vielseitigere Beobachtungen verfügen, als tatsächlich der Fall ist. Ich bevorzuge es daher mich auf eine einfache Beschreibung zu beschränken. 